\title{
Research on Expected Return Based on Time Series Model: Taking Kweichow Moutai Co., Ltd. as an Example
}

\author{
Wennuan Fang*
}

Zhejiang University of Finance \& Economics Dongfang College, Haining City, Jiaxing City 314400, Zhejiang Province, China. E-mail: 1084854008@qq.com

\begin{abstract}
With the stable development of China's economy, the economic activities among enterprises are more diversified, and the enterprise value evaluation index system is more perfect. As an important parameter in the enterprise value evaluation index, the expected income can be used to measure the profit quality of the enterprise. In order to explore the expected return of enterprises, this paper selects free cash flow as the specific index, and takes Kweichow moutai Co., Ltd. as an example, analyzes the earnings trend of enterprises through the method of time series. Time series prediction models are constructed to provide the basis for enterprise value evaluation. At the same time, by fitting single linear regression model and Autoregressive Integrated Moving Average model, the free cash flow is predicted, and finally the ARIMA $(1,2,2)$ model is obtained. The results show that the single linear regression model has a higher error rate, while ARIMA $(1,2,2)$ has a better fitting degree and a lower error rate. It can be used for the results of expected earnings of enterprises and provides a reference for enterprise value evaluation.
\end{abstract}

Keywords: Time Series Model; Expected Return; Free Cash Flow

\section{Introduction}

In recent years, with the prosperity and development of China's market economy and the continuous improvement of capital market structure, economic activities among enterprises like mergers and acquisitions, restructuring and equity transfer have become frequent, diversified and integrated. Correspondingly, the relevant index system of enterprise value evaluation is also improving day by day. At present, the methods of evaluating and estimating enterprises in China mainly include the present value of income method, the market comparison method and the cost method, among which the expected income is one of the three major parameters of the income method. The reasonable evaluation and prediction of the expected income value is conducive to the determination of the enterprise value, meanwhile, helps the enterprise managers to formulate the enterprise development strategies. The free cash flow, as the expected income index, is developed from the profit index and the net cash flow index, which can avoid the influence of different accounting modes on the evaluation results. At the same time, the free cash flow of the enterprise shows to a certain extent that the enterprise can bring the maximum cash return to shareholders and owners, and can better measure the profit quality of the enterprise. Based on this, this paper selects the single linear regression model and the Autoregressive Integrated Moving Average model (ARIMA) in the time series model, and takes Kweichow Moutai Co., Ltd. as an example to predict its expected income with free cash flow as the specific index. By comparing the two time series models, the optimal solution is obtained to provide some basis for enterprise value evaluation.

Copyright (C) 2020 Wennuan Fang

doi: $10.18282 /$ ff.v9i2.858

This is an open-access article distributed under the terms of the Creative Commons Attribution Non-Commercial License

(http://creativecommons.org/licenses/by-nc/4.0/), which permits unrestricted non-commercial use, distribution, and reproduction in any medium, provided the original work is properly cited. 


\section{Data source and interpretation of the indicator}

\subsection{Data sources}

In order to explore the expected earnings of enterprises, this paper selects free cash flow as the main indicator, obtains the free cash flow data of 18 accounting years in the consolidated statements of Kweichow Moutai Co., Ltd. from 2002 to 2019 through China Stock Market Accounting Research (SCMAR), based on which a time series model is established to predict the free cash flow of the company.

\subsection{Interpretation of the indicator}

The free cash flow of an enterprise refers to the cash flow that can pay all creditors (creditors and shareholders) after deducting taxes, necessary capital expenditure and increase of working capital. Free cash flow of enterprises is based on income and payment realization system, which can better evaluate the profit quality of the enterprise; the calculation of the free cash flow of the enterprise can exclude the abnormal income, which can objectively reflect the true profitability of the enterprise; the free cash flow of the enterprise reflects the time cost of the capital, not blindly pursuing the short-term benefits, but focusing on the long-term stable and balanced development of the enterprise, which is more conducive to measuring the true development of the enterprise prospects. The free cash flow data of Kweichow Moutai Co., Ltd. is as follows:

\begin{tabular}{c|c|c|c|c|c}
\hline Year & $\begin{array}{c}\text { Free cash flow of enterprise } \\
\text { (unit: yuan) }\end{array}$ & Year & $\begin{array}{c}\text { Free cash flow of } \\
\text { enterprise (unit: yuan) }\end{array}$ & Year & $\begin{array}{c}\text { Free cash flow of enterprise } \\
\text { (unit: yuan) }\end{array}$ \\
\hline 2002 & -62003810.8 & 2008 & 247432399.7 & 2014 & 6100741591 \\
\hline 2003 & -7053100.608 & 2009 & 746246551.6 & 2015 & 7265657933 \\
\hline 2004 & 205215256.3 & 2010 & 1037207996 & 2016 & 9613498194 \\
\hline 2005 & 178740511.7 & 2011 & 2074041420 & 2017 & 8451375592 \\
\hline 2006 & 685566027.7 & 2012 & 1566239355 & 2018 & 15646290353 \\
\hline
\end{tabular}

Table 1. Free cash flow statement of Kweichow Moutai Co., Ltd. from 2002 to 2019

\section{Introduction of the model and correlation analysis of time series}

\subsection{Introduction of the model}

\subsubsection{Single linear regression prediction model}

The main idea of single linear regression model is based on the least square method to determine the minimum sum of deviation squares of the regression equation, and to build a single regression equation as follows:

$$
y_{n}=a+b n+c
$$

Among them, the constant $a$ is the intercept of single linear regression trend line, and the constant $b$ is the slope of trend line equation, all of which are measured by least square estimation. Term $\mathrm{c}$ is a normal distribution satisfying the mean value of 0 and the homogeneity of variance. The goodness of fit of trend line is tested by hypothesis, and the final prediction is made.

\subsubsection{Autoregressive Integrated Moving Average model (ARIMA)}

Autoregressive Integrated Moving Average model can be regarded as the sum of two time series models, vector autoregressive model and moving average model. When modeling ARIMA ( $p, d, q)$ model, $p$ and q represent the order of autoregressive lag and the order of moving average, respectively. $d$ is the number of difference items needed to become a stationary time series. The prediction equation is as follows:

$$
Y_{n}=A R_{1} Y_{n-1}+A R_{2} Y_{n-2}+\ldots+A R_{n} Y_{t-n}+M A_{1} Y_{n-1}+M A_{2} Y_{n-2}+\ldots+M A_{n} Y_{t-n}+C_{n}
$$


In the equation, $A R_{1} \ldots A R_{n}$ is the autoregressive lag coefficient, $M A_{1} \ldots M A_{n}$ is the moving average item family array, $C_{n}$ is the constant residual term of the equation. The observation value needs to meet the requirements of stationary time series, and the final model prediction needs to pass the residual white noise test.

\subsection{Correlation analysis of time series}

In order to have a more intuitive understanding of the changes of free cash flow of enterprises in Kweichow Moutai Co., Ltd. in 18 years, and facilitate further analysis of free cash flow of enterprises, relevant figures are drawn according to the data, and the results are shown in Figure 1 below:

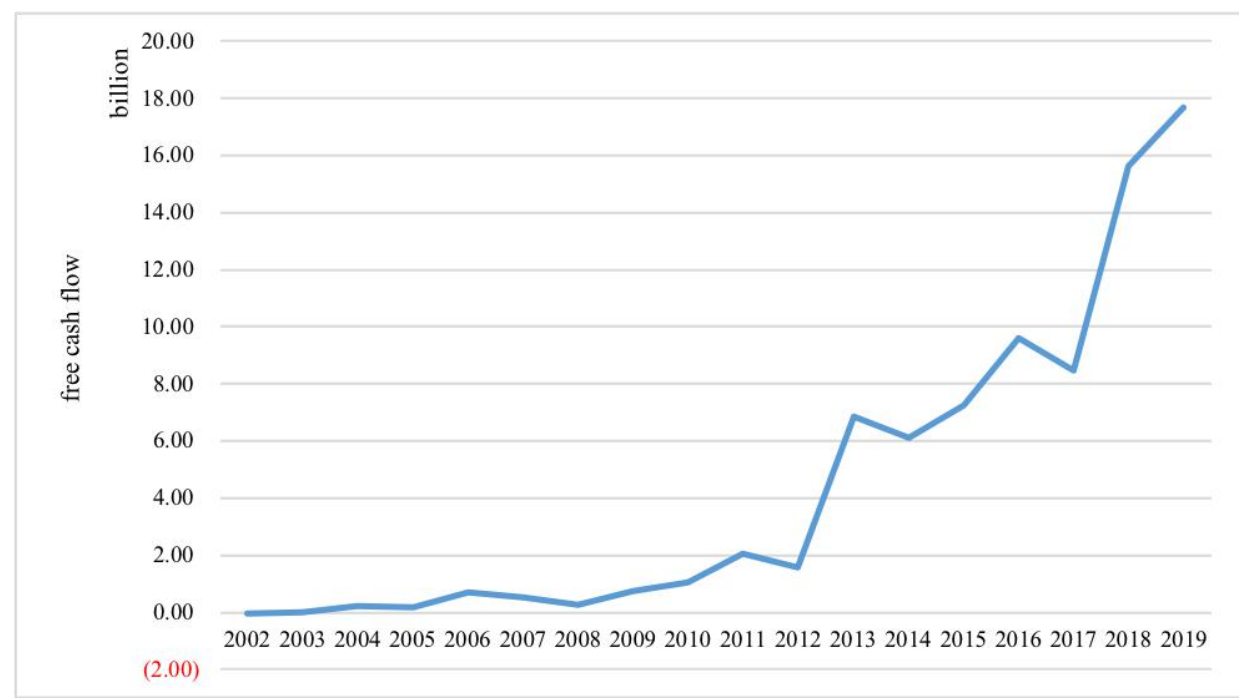

Figure 1. Change trend of free cash flow of enterprises in Kweichow Moutai Co., Ltd. from 2002 to 2018.

Based on the analysis of the change of free cash flow of enterprises in Kweichow Moutai Co., Ltd. from 2002 to 2019 , the trend of its free cash flow was generally in line with the development trend of the whole liquor industry and macro industrial policies. 2002 to 2012 were the golden period for the development of Kweichow Moutai Co., Ltd. It has maintained the development strategy of expansion, with more capital expenditure. The fluctuation of free cash flow in the early stage is mainly reflected in the expansion of production line and the new construction of logistics and storage workshop. At the same time, the government issued eight regulations restricting government consumption in 2012, slowing down the growth of free cash flow in 2013-2015, as Kweichow Moutai Co., Ltd. mainly produces high-end liquor. In this regard, the company has carried out strategic transformation since 2015, established the "133 brand strategy" (a world brand, three national brands and three regional brands) and the " $5+5$ " market strategy of Maoxiang series liquor (to build five core markets and five potential markets, with the sales market turning to the counties and townships), transformed from government consumption to business consumption and personal consumption, and increased middle and low-end product categories. Since 2015, the proportion of sales of high-priced Moutai liquor has declined, while the sales of low-priced series liquor has started to rise with an increasing market share. Since then, Kweichow Moutai has entered the second golden development period. In 2016-2017, as the initial goal of the strategy is to seize the market rather than make profits, the free cash flow has declined; the implementation of the strategy is stable in 2018-2019, low-priced series of liquor has a certain consumer market, and the free cash flow of enterprises has started to grow rapidly.

\section{Prediction of free cash flow of enterprises}

\subsection{Pretreatment of free cash flow of enterprises}

Since the Autoregressive Integrated Moving Average model requires the time series to meet the stationary state: 
that is to say, the trend of free cash flow in Kweichow Moutai Co., Ltd. does not contain obvious seasonal, cyclical and cyclical characteristics, and it is in an irregular fluctuation. Therefore, it is necessary to preprocess the data. The steps are: first, the processing of missing value. Because the free flow of the enterprise in 18 years is recorded from the database and is real and effective, the original sequence does not contain the missing value. Second, the smooth processing of the original data. First of all, the data is logarithmically processed; secondly, by drawing the autocorrelation graph and partial autocorrelation graph, it is found that the autocorrelation graph and partial autocorrelation graph showing an obvious tail shape, that is, there is no significant shape of 0 after a certain lag order. Therefore, the logarithm of the original free cash flow sequence is not a stationary time series. Through the unit root test, it is found that the $\mathrm{p}$ value is 0.01 less than 0.05 , rejecting the hypothesis that the unit root exists, indicating that the sequence after the second-order difference has remained stable. The time sequence diagram, autocorrelation diagram and partial autocorrelation diagram after the second-order difference are as follows:

x.ji
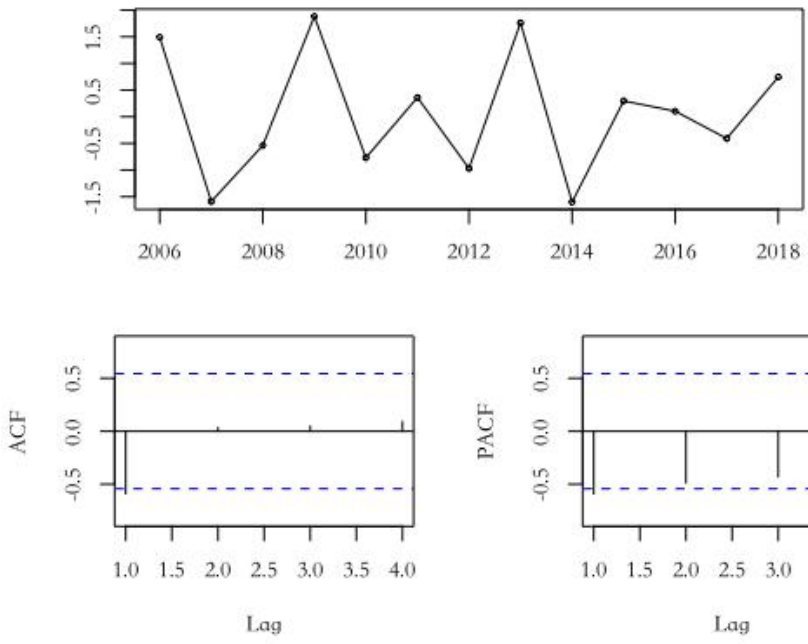

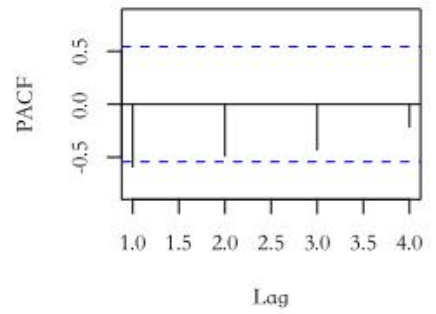

Figure 2. Sequence diagram, autocorrelation diagram and partial autocorrelation diagram after second-order difference.

At the same time, by observing the sequence diagram of free cash flow of Kweichow Moutai Co., Ltd. after the second-order difference, it is found that after the second-order difference, the difference sequence presents irregular changes; from the autocorrelation diagram and partial autocorrelation diagram, it can be seen that the autocorrelation diagram and partial autocorrelation diagram of the difference sequence show tailing, that is, the step-by-step attenuation is not significant 0 , and there is no significant trend. It can be seen that the free cash flow sequence after the second-order difference in Kweichow Moutai Co., Ltd. is a stationary time series, which can be used for time series model prediction.

In this paper, the stationary time series are obtained after the second-order difference. The ARIMA (p, 2, q) model can be established for the original series. From the second-order difference autocorrelation chart in Figure 2, it can be seen that the autocorrelation sequence chart shows a declining trend, and there is a more obvious peak, and there are two more obvious peaks in the partial autocorrelation chart, gradually decreasing to 0 . Therefore, this paper attempts to establish ARIMA $(1,2,2)$ time series model to predict the free cash flow of Kweichow Moutai Co., Ltd.

\subsection{Specific prediction and prediction results of cash flow of enterprises}

In this paper, single linear regression prediction model and Autoregressive Integrated Moving Average model are used to predict the free cash flow of Kweichow Moutai Co., Ltd. The specific prediction process is as follows:

\subsubsection{Single linear regression prediction model}


Single linear regression model has less reading value of free cash flow data of Kweichow Moutai Co., Ltd., which is easy to be affected by individual extreme value, and can not accurately reflect the expected trend of its free cash flow. In order to compare with the Autoregressive Integrated Moving Average model and reflect the accuracy of the time series model to the expected return, the single linear regression prediction results achieved in this paper are as follows:

\begin{tabular}{c|c|c|c|c}
\hline Variables & Coefficient & Standard error & T statistic & P value \\
\hline constant & -1579651764565.43 & 232630004071.308 & -6.790404234 & 0.609113 \\
\hline $\mathrm{x}$ & 787686261.4 & 115735976.7 & 6.80588944 & 0.593318 \\
\hline
\end{tabular}

Table 2. Significance level of original sequence parameters

The forecast value of free cash flow of enterprises in the next five years is as follows:

\begin{tabular}{c|c|c|c|c|c}
\hline Forecast year & $\mathbf{2 0 2 0}$ & $\mathbf{2 0 2 1}$ & $\mathbf{2 0 2 2}$ & $\mathbf{2 0 2 3}$ & $\mathbf{2 0 2 4}$ \\
\hline $\begin{array}{c}\text { Forecast value of } \\
\text { free cash flow } \\
\text { (unit: yuan) }\end{array}$ & 11474483503 & 12262169764 & 13049856026 & 13837542287 & 14625228548 \\
\hline
\end{tabular}

Table 3. Forecast value of Single linear regression of expected income of Kweichow Moutai Co., Ltd.

To a large extent, the model considers the overall change of free cash flow of Kweichow Moutai Co., Ltd., but the model is insufficient to extract the information of abnormal value, and the extreme individual value has a great impact on the model. From 2020 to 2024, the forecast value of its free cash flow began to rise rapidly from about 11.4 billion yuan to 14.6 billion yuan. This is not in line with the basis of free cash flow of nearly 17.6 billion yuan in 2019 . Even in the prediction of time series model, the single linear regression method is not suitable for predicting the expected return. With the increase of time, there will be a big gap between the predicted value and the real value, and the single linear regression model is difficult to pass the parameter hypothesis test.

\subsubsection{Autoregressive Integrated Moving Average model (ARIMA)}

In this paper, the stationary time series are obtained by the second-order difference method. The ARIMA (p, 2, q) model can be established for the logarithmic original series. From the second-order difference autocorrelation chart in Figure 2, it can be seen that the autocorrelation sequence chart shows a declining trend, and there is a more obvious peak, and there are two more obvious peaks in the partial autocorrelation chart, gradually decreasing to 0 . Therefore, the time series model of ARIMA $(1,2,2)$ is established. The $\mathrm{p}$ value of the residual white noise test is 0.5728 , which is greater than 0.05 . The received residual is the white noise series. That is to say, ARIMA $(1,2,2)$ model passes the white noise test. Therefore, the model is applicable to the prediction of free cash flow of Kweichow Moutai Co., Ltd., and the prediction results are as follows:

\begin{tabular}{c|c|c|c|c|c}
\hline Forecast year & $\mathbf{2 0 2 0}$ & $\mathbf{2 0 2 1}$ & $\mathbf{2 0 2 2}$ & $\mathbf{2 0 2 3}$ & $\mathbf{2 0 2 4}$ \\
\hline $\begin{array}{c}\text { Forecast value } \\
\text { of free cash } \\
\text { flow (unit: } \\
\text { yuan) }\end{array}$ & 27150193218 & 36226618382 & 40052084991 & 30120906497 & 57317173931 \\
\hline
\end{tabular}

Table 4. Predicted value of ARIMA model of expected return of Kweichow Moutai Co., Ltd.

\subsection{Error analysis of the prediction results of time series models}

Based on the true value of free cash flow of Kweichow Moutai Co., Ltd. in 2019, the error of the two time series models is compared and analyzed. The results are as follows: 


\begin{tabular}{c|c|c|c}
\hline Model & predicted value & True value & Error rate \\
\hline $\begin{array}{c}\text { Single linear regression } \\
\text { model }\end{array}$ & 10686797241 & 17690662346 & $39.59 \%$ \\
\hline ARIMA model & 18768579399 & 17690662346 & $6.09 \%$ \\
\hline
\end{tabular}

Table 5. Error comparison of free cash flow for Kweichow Moutai Co., Ltd. in 2019

By comparing the real value of free cash flow of Kweichow Moutai Co., Ltd. with the predicted value in 2019, it can be found that the error rate of single regression model is as high as $39.59 \%$, which shows that the model is not suitable for the prediction of free cash flow of enterprises; the error rate of autoregressive moving average model is low, only $6.09 \%$, which is a good fit for the prediction of free cash flow of Kweichow Moutai Company.

\section{Conclusion}

This paper studies the prediction of the expected return index under the method of benefit present value in the enterprise value evaluation, and select the free cash flow of the enterprise as the specific index. Taking Kweichow Moutai Co., Ltd. as an example, a single linear regression model and the Autoregressive Integrated Moving Average model are established. After comparative analysis, it has been found that the Autoregressive Integrated Moving Average model has a better fitting degree and a lower error rate, and the measured value of the final prediction can better reflect the operation status of Kweichow Moutai Co., Ltd.Therefore, the Autoregressive Integrated Moving Average model has a certain guiding significance for the prediction of the expected return index. However, it still has some shortcomings. Therefore, when evaluating the value of an enterprise, an appropriate model should be selected to predict the actual business situation of the enterprise.

\section{References}

1. Ma X, Cai R, Ning Q, et al. State estimation of time series NAR model based on auxiliary particle filter and grey prediction (in Chinese). Statistics \& Decision 2019; 35(4): 27-31. doi: 10.13546/j.cnki.tjyjc.2019.04.005.

2. Yang H, Pan Z, Bai W. A review of time series prediction methods (in Chinese). Computer Science 2019; 46(1): 21-28.

3. Pan H, Zhang C. FEPA-financial time series adaptive combinatorial prediction model (in Chinese). Chinese Journal of Management Science 2018; (6). doi: 10.16381/j.cnki.issn1003-207x.2018.06.004.

4. Ma S, Fan Y. Policy evaluation of China's new energy vehicles based on time series co-integration (in Chinese). China Population, Resources and Environment 2018; 28(4): 117-124.

5. Yan Y. GDP analysis and forecast of Shandong Province based on ARIMA model (in Chinese). Mathematics in Practice and Theory 2018; (4).

6. Jiang X. An empirical analysis of the reasons for the deviation of RMB internal and external value based on VAR model (in Chinese). Statistics \& Decision 2018; 34(4): 157-160. doi: 10.13546/j.cnki.tjyjc.2018.04.035.

7. Wang Z, Xu G. Research on enterprise value evaluation model embedded in CSR behavior (in Chinese). East China Economic Management 2017; 31(11): 137-142. doi: 10.3969/j.issn.1007-5097.2017.11.018.

8. Chen Z, Tan Y, Lu H. Firms' patent signals and value assessment: Based on venture capital's view. Shanghai Economic Review 2017; (10): 105-114.

9. Hu Z. The time gap between fiscal investment and regional innovation output: The analysis of VAR model based on time series data. Economic Survey 2017; 34(3): 141-146.

10. Ma Z, Han Z. Study on complex seasonal time series model. Statistics \& Decision 2017; (6): 29-32. doi: 10.13546/j.cnki.tjyjc.2017.06.006. 